and demographic and occupational characteristics of healthcare providers. The findings have important implications for further training of specific healthcare providers with considerations to their ethnic belongings.

\title{
0322 SCREENING FOR INTIMATE PARTNER VIOLENCE IN HEALTHCARE IN KANO, NIGERIA: EXTENT AND DETERMINANTS
}

I A John*, S Lawoko, L Svanström Correspondence: Karolinska Institute, Department of Public Health Sciences, Division of Social Medicine, Norrbacka Plan 2, Stockholm, 17176, Sweden

10.1136/ip.2010.029215.322

Introduction The adverse health consequences of domestic violence have led to increased call to involve healthcare providers in the management of partner violence prevention through screening for it within the healthcare.

Objective To assess the extent and determinants of screening for Intimate Partner Violence (IPV) at Aminu Kano Teaching Hospital, Kano, Nigeria.

Method 274 healthcare providers responded to the domestic violence healthcare provider survey probing the frequency of screening for IPV, staff attitudes towards domestic abuse, perceived self efficacy in screening, availability of support networks and staff/patient safety in regard to IPV inquiry. t-Test and logistic regression were employed to study determinants of screening.

Results Majority of participants (74\%) had not screened for IPV during the preceding 3 months. Male gender, being elderly and of Yoruba ethnicity increased the likelihood of screening. With increasing perceived efficacy and increasing blame of the victim for abuse, the likelihood of screening for IPV increased.

Conclusion Barriers to effective screening of IPV may emanate from the attitudes of healthcare provider towards IPV 